INPLASY

PROTOCOL

To cite: Zhao et al. High

Prevalence of Thyroid

Carcinoma in Patients with

Insulin Resistance: A Meta-

analysis of Case-control

Studies. Inplasy protocol

202180043. doi:

10.37766/inplasy2021.8.0043

Received: 11 August 2021

Published: 11 August 2021

Corresponding author:

Lin Liao

liaolin009@sina.com

Author Affiliation:

Department of Endocrinology and Metabology, The First Affiliated Hospital of Shandong First

Medical University \& Shandong

Provincial Qianfoshan Hospital,

Ji-nan 250014, China

Support: ZR2019PH025.

Review Stage at time of this submission: Preliminary searches.

\section{High Prevalence of Thyroid Carcinoma in Patients with Insulin Resistance: A Meta-analysis of Case- control Studies}

\author{
Zhao, JY1; Zhang, QP2; Yang, YP3; Yao, JM4; Liao, L5; Dong, JJ6.
}

Review question / Objective: The association between insulin resistance and thyroid carcinoma is controversial. We conducted this meta-analysis of association between insulin resistance and thyroid carcinoma.

Information sources: The following databases: PubMed, Cochrane Library, Sinomed, CNKI and Wanfang, for casecontrol studies of insulin resistance in thyroid carcinoma were searched. We also searched the additional trials at the clinical trial register centers (http://www.ClinicalTrials.gov). The literature search for this meta-analysis was restricted to published results. Databases were searched from the earliest data to 1 February, 2021, published in English or Chinese language.

INPLASY registration number: This protocol was registered with the International Platform of Registered Systematic Review and Meta-Analysis Protocols (INPLASY) on 11 August 2021 and was last updated on 11 August 2021 (registration number INPLASY202180043).

Conflicts of interest:

None declared.

\section{INTRODUCTION}

Review question / Objective: The association between insulin resistance and thyroid carcinoma is controversial. We conducted this meta-analysis of association between insulin resistance and thyroid carcinoma.

Condition being studied: Markedly increased incidence of thyroid carcinoma has been reported over the past decades all over the world. The yearly incidence of thyroid carcinoma tripled from 4.9 per 
100000 to 14.3 per 100000 during the past three decade in the United States. Moreover, the collection of cancer registration data of China in 2013 showed that the incidence in female reached $\mathbf{1 6 . 3 1}$ per 100000 and became the fifth most frequent cancer. This increment can be ascribed to the rapid development and widely use of sonography techniques. However, it can not totally explan the increase incidence of macrocarcinoma. Nowadays, the known risk factors for thyroid carcinoma include the head and neck radioactive exposure, female, advanced age, iodine deficiency or excessive and family history of thyroid carcinoma. Unfortunately, the definately mechanisms of these risk factors were not clearly illuminated and these also cannot explain the increased incidence totally. More importantly, most of the known factors are ineluctable. Recently, some scientists proposed that insulin resistance or hyperinsulinemia might be another potential risk factor for thyroid carcinoma.

\section{METHODS}

Participant or population: Eligible studies met the following criteria: (1) study assessed the association between insulin resistance or hypersinulinemia and thyroid carcinoma; (2) study was designed as the case-control study; (4) study reported at least one of the following three outcomes: the fasting serum insulin level, HOMA-IR and the incidence of insulin resistance in patients with thyroid carcinoma.

Intervention: Higher level of the fasting serum insulin and HOMA-IR, with the incidence of insulin resistance

Comparator: Normal levels of fasting serum insulin and HOMA-IR, without the incidence of insulin resistance.

Study designs to be included: Case-control studies.

Eligibility criteria: Eligible studies met the following criteria: (1) published in English or Chinese language; (2) study assessed the association between insulin resistance or hypersinulinemia and thyroid carcinoma; (3) study was designed as the case-control study; (4) study reported at least one of the following three outcomes: the fasting serum insulin level, HOMA-IR and the incidence of insulin resistance in patients with thyroid carcinoma.

Information sources: The following databases: PubMed, Cochrane Library, Sinomed, CNKI and Wanfang, for casecontrol studies of insulin resistance in thyroid carcinoma were searched. We also searched the additional trials at the clinical trial register centers (http:// www.ClinicalTrials.gov). The literature search for this meta-analysis was restricted to published results. Databases were searched from the earliest data to 1 February, 2021, published in English or Chinese language.

Main outcome(s): Association between insulin resistance and thyroid carcinoma.

Quality assessment / Risk of bias analysis: Two reviewers independently assessed the Newcastle-Ottawa Scale (NOS) for casecontrol studies. A study can be scored one star for each numbered item within the Selection and Exposure categories, and two stars can be given for Comparability. The score of nine is the highest and shows the highest quality. Disagreements were resolved by evaluating and discussing between two reviewers.

Strategy of data synthesis: The outcomes include the level of fasting serum insulin, HOMA-IR, the incidence of insulin resistance in patients with thyroid cancer. Fixed-model or random-model was performed by weighted mean difference (WMD), standardized mean difference (SMD) and $95 \%$ confidence intervals (Cl) for continuous variables. Fixed-model performed by computing odds ratio (OR) and $95 \% \mathrm{Cl}$ for dichotomous variables.

Subgroup analysis: None.

Sensitivity analysis: The 12 was calculated as an index of heterogeneity between studies. The analyses were performed by 
Review Manager 5.3 (Cochrane Collaboration, United Kingdom, http:// www.cochrane.org) and STATA.

Country(ies) involved: China.

Keywords: insulin resistance, HOMA-IR, thyroid carcinoma, meta-analysis, casecontrol study.

Contributions of each author:

Author 1 - Junyu Zhao.

Email: 13589066435@163.com

Author 2 - Qianping Zhang.

Author 3 - Yupeng Yang.

Author 4 - Jinming Yao.

Author 5 - Lin Liao.

Author 6 - Jianjun Dong. 